\title{
Title: A comparison of the effects of different stretching methods on flexibility, muscle activity, and pain threshold in ballet dancers; a preliminary randomized controlled trial.
}

\author{
Min Gyun $\mathrm{Ko}^{1}$, Chang Ho Song ${ }^{1 *}$, Myung Mo Lee ${ }^{2}$ \\ ${ }^{1}$ Department of Physical Therapy, Sahmyook University, 26-21 Gongneung2-dong, Nowon-gu, Seoul 139-742, \\ Republic of Korea; bluesea05@hanmail.net (Ko, M.M.); chsong@syu.ac.kr (Song, C.H.) \\ ${ }^{2}$ Department of Physical Therapy, Daejeon University, 2421, Applied Science building, 62, Daehak-ro, Dong-gu, \\ Daejeon city, Republic of Korea, 34520; mmlee@dju.kr (Lee M.M.) \\ *Correspondence; chsong@syu.ac.kr; Telephone 82-2-3399-1630; FAX 82-2-3399-1638
}

\begin{abstract}
Objective: The purpose of this study is to compare the effects of stretching methods for flexibility, muscle activation, and pressure pain threshold in ballet dancers, and to suggest an effective stretching method. Methods: Thirty-three ballet dancers were randomized to a static stretching group $(n=11)$, muscle energy technique stretching group $(n=11)$, and vibrationassisted stretching group $(n=11)$. The angle of hip joint extension in arabesque, the activation of rectus femoris in Devéloppé, and the pressure pain threshold on rectus femoris in sitting position were measured to compare the effects of different stretching methods. Results: Hip joint extension angles increased in all stretching methods $(p<0.05)$, however, vibration-assisted stretching and muscle energy technique stretching were more effective than static stretching $(p<0.05)$. The activation of the rectus femoris decreased in all groups $(p<0.05)$, but the muscle energy technique stretching group and the vibration-assisted stretching group showed a significant decrease compared to the static stretching group $(p<0.05)$. The pressure pain threshold showed significant improvement only in the static stretching group $(p<0.05)$, and the vibration-assisted stretching group $(p<0.05)$. Conclusion: Vibration-assisted stretching compared to static stretching and muscle energy technique stretching is a beneficial method for flexibility, muscle activation, and pressure pain threshold in ballet dancers.
\end{abstract}

Key words: Dancing, Muscle stretching exercise, Pliability 


\section{Introduction}

Ballet is a performance art, beautifully expressing the shaping of human feelings and emotions into physiological and mechanical body movements [1]. The ability of postural control in ballet is more important than in any other sports because there should be no misalignment in the ballet dancer's body during the expression in both static and dynamic movements [2]. Dancers should have physical factors such as a well-balanced flexibility, muscular strength and endurance, and elegant and complex movements to accurately and completely perform a successful ballet performance [3].

Ballet movements include the more focal motion of the lower body than the upper body. There is always a risk of injury since ballet is performed at a high level of movements including jumping, landing, and rotating over the acceptable anatomical and physiological range with weight bearing [4]. Dancers pay special attention to physical movements and the development of the body over the normal range to overcome the risk of injury and to satisfy the demands of an art form [5].

Strength and flexibility are two important physical factors in dancers that describe the perfect ballet movement. Strength is influenced by joint angle, the length of the muscle, muscle size, number and types of active motor unit, and the movement speed of the muscle. The maximum strength of a muscle occurs when it is stretched to approximately $20 \%$ less than the length at steady state [6]. Therefore, long-term flexibility training not only helps to improve muscle strength, but also improves performance [7]. Also, one essential factor to help prevent unexpected injuries is flexibility. Flexibility is an important component of physical fitness usually included in general exercise programs [8].

The promotion of flexibility is accomplished through correct stretching exercises. Widely known stretching methods are static stretching, ballistic stretching, passive stretching, active 
stretching, and muscle energy technique (MET) stretching. However, the efficiencies of stretching methods exhibit different results for each researcher. MET stretching is used to promote relaxation after maximal isometric contraction against the static resistance of the stretched muscle by proprioceptive neuromuscular facilitation, and to increase the range of motion and flexibility of the related muscles [9]. Some studies report MET stretching as more efficient than both static and elastic stretching [10]. However, in another study, there were no clinically relevant differences in the effects of stretching among a single static, ballistic, or PNF stretching techniques [11]. However, the various results are difficult to generalize in a particular subject due to various methodological differences, such as training status at baseline, the dosage of the intervention, stimulus duration, site of application, and the participant. Despite the previous studies on stretching, stretching methods in ballet training have not been supported by scientific evidence. Moreover, there is not enough research regarding strategic stretching methods despite the fact that professional flexibility training may express skilled ballet movements with the potential possibility of improvement [12].

Stretching techniques combined with localized vibration stimulation, shows a greater effect on the improved range of motion more than stretching techniques alone; this has recently gained interest by researchers $[13,[14]$. The stretching technique combined with vibration stimulation uses a safe vibration to increase bone density, resulting in the decrease of abnormal muscle contraction due to quick contraction and the relaxation of the muscles. In addition to this, it stimulates the Golgi tendons making it an efficient method of muscle relaxation while simultaneously increasing the range of motion [15].

Therefore, the aim of this study is to implement static stretching, MET stretching, and vibration assisted (V-A) stretching methods in ballet dancers to compare and examine the flexibility of the rectus femoris (RF), muscle activation, and pain, and suggest an effective 
stretching method. We hypothesize that the V-A stretching technique is more effective in flexibility, muscle activation, and pain in ballet dancers compared to the other stretching methods. 


\section{Materials and methods}

\section{Participants}

Forty-two ballet dancers, who are members of U ballet company in Seoul, were recruited according to the following inclusion criteria: (1) practice more than 15 hours and less than 17 hours a week on average, and (2) trained consistently without a break of 1 month or longer. The exclusion criteria included: (1) having neurological or orthopedic diseases, (2) taking medication for muscle relaxation, (3) unable to undergo vibration stimulation due to pregnancy or nephrolith thrombus infectious diseases, and (4) those who participated in a similar study within a year. This study was conducted according to the ethical requirements of the Institutional Review Board of Sahmyook University for human experimentation (SYUIRB2014-110) and to the Helsinki Declaration. Also, the protocol was approved by the International Clinical Trials Registry Platform of World Health Organization (permit number: KCT0003071). All study participants signed a statement of informed consent.

\section{Procedures}

They were randomly assigned to static stretching group, MET stretching group, and V-A stretching group. The randomization process was performed using computer-generated numbers produced by a basic random number generator (random allocation software version 1.0; University of Medical Sciences, Iran, 2004) [16]. Before the interventions, all of the participants were assessed for basic abilities including maximum voluntary isometric contraction (MVIC) of the RF. Prior assessments included hip joint extension angle during arabesque, RF activation during Devéloppé, and pressure pain threshold of the RF. All assessments and interventions were administered only on the ballet dancers' dominant side.

Participants performed static stretching in the prone position with a posterior pelvic tilt. 
While maintaining this position, the participants pulled on their ankles with their hand until the knee was flexed parallel to the floor. Participants maintained this position until they felt a strong tension in the RF (Figure. 1-a). Participants performed MET stretching in a prone position with resistance applied by the assessor in the knee flexion and hip extension direction (maximum without pain). Participants contracted the RF and maintained the position for 10 seconds before passively extending to 10 seconds (Figure. 1-b). Participants performed V-A stretching by applying focal vibration on the muscle belly of the RF when it was highly contracted. To apply the focal vibrator (Turbo Saso; Youngil M (Inc.), Korea. 2009) on the RF, diffused sponge-type tool onto the vibrator and fixed it with a strap (Figure. 1-c). To obtain an effective stretching effect, vibration stimulation of $30 \mathrm{~Hz}$ was administered. All stretching were repeated for 4 sets, 30 seconds per set, and a 30 second break was given between each set. The participants were sufficiently informed about the intervention methods, and all participants received a post-test stretching after 10 minutes using the same pre-test method. One physical therapist, blinded to the treatment allocation, performed the application of the focal vibrator and all assessments (Figure 2). 
Figure 1. The position according to the stretching methods.

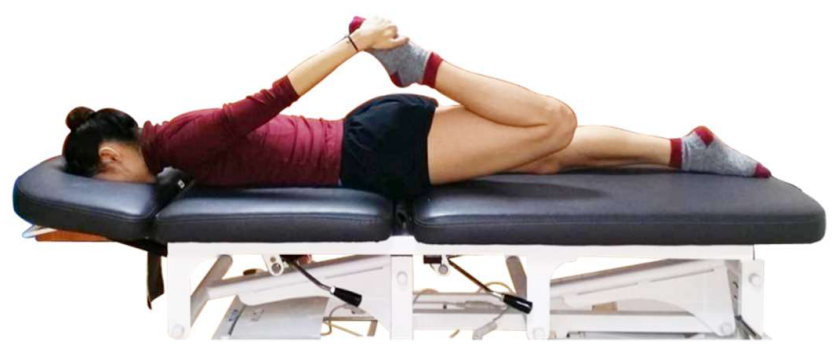

a. Static stretching method

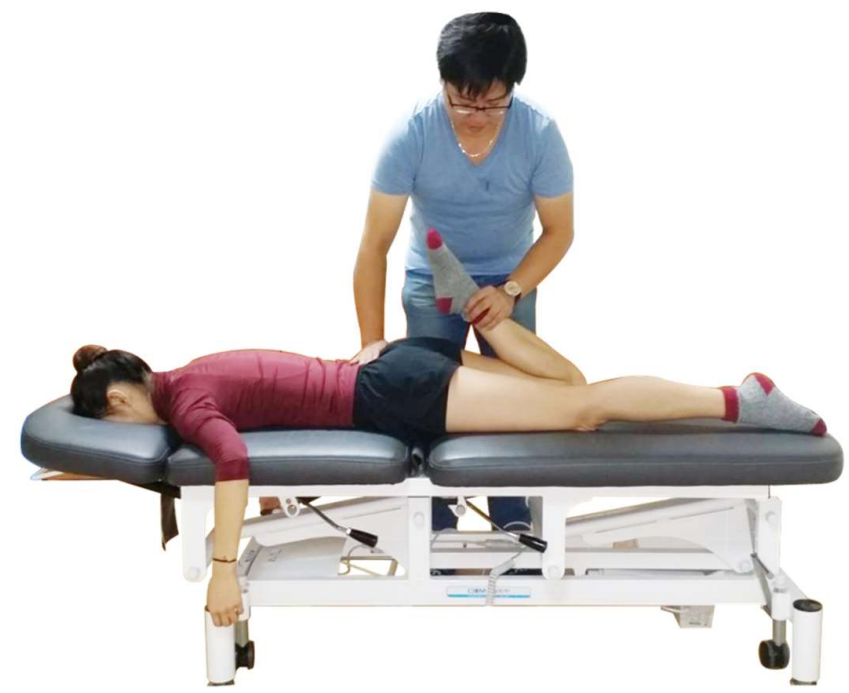

b. MET stretching method

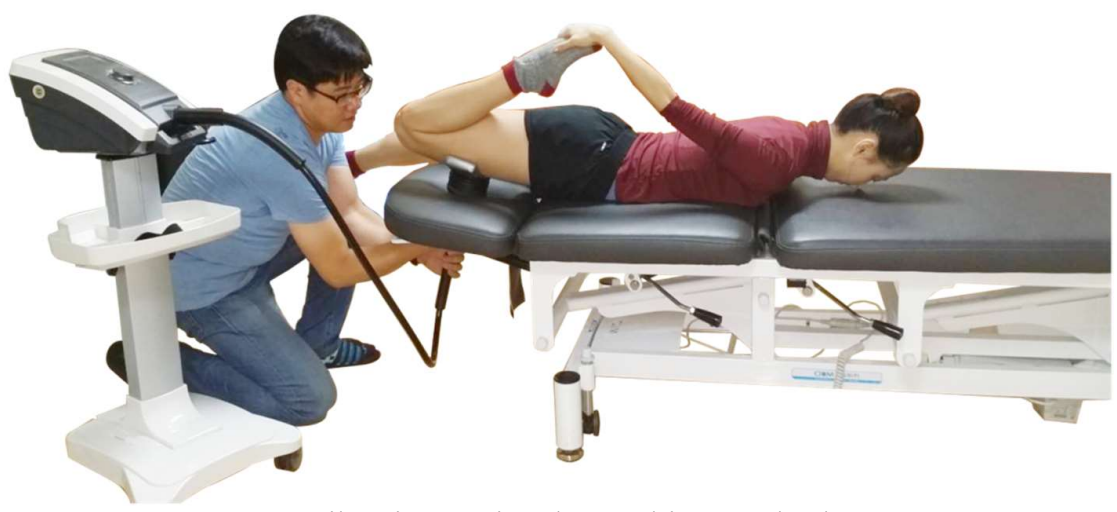

c. Vibration-assisted stretching method 
Figure 2. CONSORT Flow chart of the study.

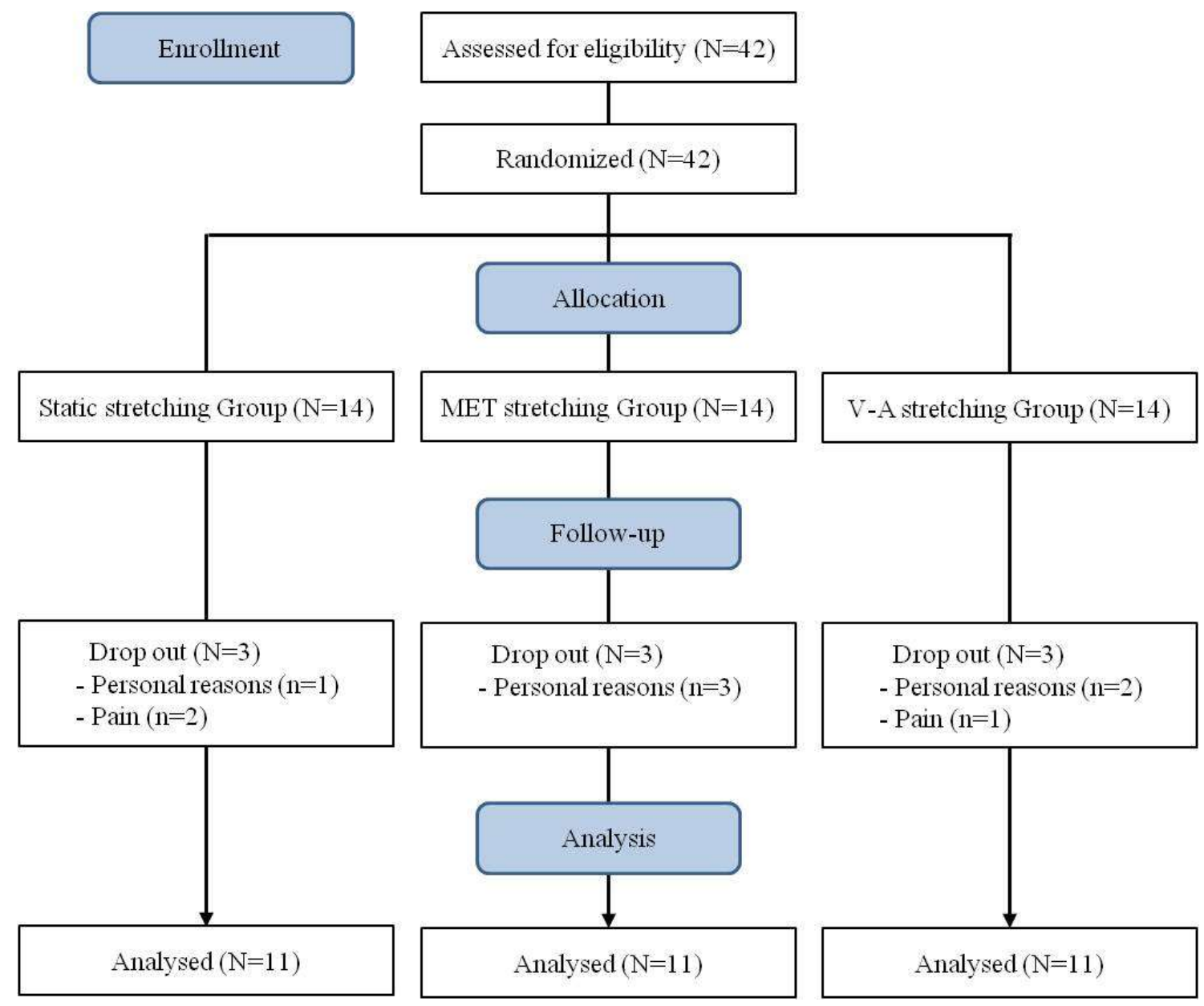




\section{Outcome measurements}

The effects of each stretching methods were compared through flexibility, muscle activation, and pain threshold. To compare the flexibility of the RF in different stretching methods, the angle of hip extension while in arabesque was measured. Arabesque is a movement where the person erects the upper body and the knee opposite the pivot leg is completely extended to lift that leg behind the body as high as possible. Arabesque requires hip flexibility, especially the flexibility of the RF. To minimize compensation arising in the pelvis and lower back before the measurement, the participants laid in the prone position on the table fixing their pelvis. They performed the maximal arabesque with only the extension of the lower body, at this moment their pelvis extension angles were measured.

Devéloppé is a ballet movement in which one leg is raised to the knee of the supporting leg and fully extended. Since Devéloppé is a movement including hip flexion and knee extension, it is highly related to the activation of the RF, which is the main muscle for hip flexion and knee extension. For the measurement of RF activation while in Devéloppé, participants maintained parallel pelvic alignment with their legs fully extended after fixing the upper body against a wall to prevent lordotic lumbar flexion. Surface wireless Trigno (Trigno Wireless EMG system; Delsys Inc., USA, 2012), which collects joint angle and muscle contraction signals simultaneously, was used to measure hip extension angle in arabesque and RF activation in Devéloppé. Trigno sensor was attached midway between the base of patella and anterior superior iliac spine (ASIS), in which this is the midpoint of the muscle during isometric contraction of the lower limb. To minimize the skin resistance, the sensors were wiped with rubbing alcohol and dried off. To attach the sensor in the same location, the location was marked with the experimental pen. The data collected through Trigno sensor was analyzed through EMG works Analysis after processing the wirelessly transmitted electromyogram 
signals to the Trigno Base Station with Delysis EMG works 4.1.1 (Delsys Inc., USA, 2012) software. The sampling rate to obtain EMG signals was $1000 \mathrm{~Hz}$, where this was band-pass filtered within the range of $20 \sim 450 \mathrm{~Hz}$. After undergoing the full wave rectification process, it was processed with RMS (root mean square) and analyzed. To normalize the EMG value of the RF, MVIC was measured for 5 seconds in manual muscle testing position before the pretest. A 2-minute break was given between the measurements totaling 3 trials. Excluding the first and last 1 seconds of the 5 second measurement value, the data were processed with RMS and made the maximum EMG signal as $100 \%$ MVIC.

Digital Algometer Commander ${ }^{\mathrm{TM}}$ (JTECH Medical, Japan, 2007) was used to measure pressure pain of RF by different stretching methods. While sitting with the upper body in an upright position, Algometer was administered vertically at the midpoint between the base of the patella and the ASIS, where the surface EMG was attached. Researchers asked the participants to indicate when they felt pain, and recorded the pressure value at that point. Three consecutive trials were made and the mean value was used as the pain threshold value.

\section{Statistical analysis}

All Statistical analyses were performed using SPSS version 19.0 statistical software (SPSS Inc., Chicago, IL, USA). Results are presented as the mean \pm standard deviation (SD). Prior to training, the Shapiro-Wilks test was used to assess the normality. One-way analysis of variance was used to assess differences in the normally distributed continuous variables, and the Scheffé post hoc test to examine the differences. Paired t-test was used to assess differences in continuous variables within groups before and after interventions for each group. Post hoc analyses were performed using Scheffé test. Differences in categorical variables were analyzed using $\chi^{2}$ test. Statistical significance was set below 0.05 for all $p$ values. 


\section{Results}

Forty-two participants were randomized to a static stretching group $(n=14)$, MET stretching group ( $n=14)$, and V-A stretching group $(n=14)$. Three participants in each group dropped out due to personal reasons and/or sudden pain. Thus, each group contained 11 participants with participants completing the study (Figure. 2). There were no significant differences in the general characteristics and the dependent variables group at baseline between the static stretching group, the MET stretching group, and the V-A stretching (Table 1).

The change in the Arabesque angle after the intervention showed a significant improvement from $40.87^{\circ}$ to $41.83^{\circ}$ in the static stretching group $(p<0.05), 36.39^{\circ}$ to $39.19^{\circ}$ in the MET stretching group $(p<0.05)$, and $31.32^{\circ}$ to $37.08^{\circ}$ in the $\mathrm{V}$-A stretching group $(p<0.05)$. Across the three groups, the degree of change in the Arabesque angle was statistically greater in the MET stretching group and the V-A stretching group than in the static stretching group $(p<0.05)$.

The change in muscle activation during Devéloppé according to the intervention showed a significant decrease from $69.90 \%$ to $63.75 \%$ in the static stretching group $(p<0.05), 65.03 \%$ to $52.45 \%$ in the MET stretching group $(p<0.05)$, and $67.24 \%$ to $56.14 \%$ in the V-A stretching group $(p<0.05)$. When comparing the three groups, the degree of change in muscle activation was statistically greater in the MET stretching group and the V-A stretching group than in the static stretching group $(p<0.05)$.

The pressure pain threshold according to the intervention showed significant improvement from $25.13 \mathrm{~N}$ to $26.88 \mathrm{~N}$ in the static stretching group $(p<0.05)$, and $19.18 \mathrm{~N}$ to $21.79 \mathrm{~N}$ in the VA stretching group $(p<0.05)$. However, there were no significant improvements in the MET stretching group. In addition, pre-and post-intervention differences were not significant (Table $1)$. 
Table 1. General characteristics of the participants, and the changes in the dependent variables according to the stretching methods.

\begin{tabular}{lcccc}
\hline \hline Variables & $\begin{array}{c}\text { Static stretching } \\
\text { Group(A) }(n=11)\end{array}$ & $\begin{array}{c}\text { MET stretching } \\
\text { Group(B) }(n=11)\end{array}$ & $\begin{array}{c}\text { V-A stretching } \\
\text { Group }(\mathrm{C})(n=11)\end{array}$ & $\begin{array}{c}\mathrm{F}^{\dagger}(p) \\
\text { Post hoc }\end{array}$ \\
\hline Age(years) & $27.45 \pm 3.59^{\mathrm{a}}$ & $26.90 \pm 3.67$ & $28.54 \pm 3.56$ & $0.587(0.562)$ \\
Height(cm) & $174.82 \pm 6.94$ & $171.74 \pm 6.31$ & $170.57 \pm 6.21$ & $1.275(0.294)$ \\
$\begin{array}{l}\text { Weight(kg) } \\
\begin{array}{l}\text { Work Experience } \\
\text { (years) }\end{array}\end{array}$ & $57.73 \pm 12.32$ & $53.00 \pm 9.62$ & $53.27 \pm 8.14$ & $0.748(0.482)$ \\
& $13.55 \pm 3.08$ & $14.55 \pm 4.06$ & $14.45 \pm 3.05$ & $0.287(0.753)$
\end{tabular}

The angle of hip joint extension in arabesque

$\begin{array}{lcccc}\text { Pre } & 40.87 \pm 6.96 & 36.39 \pm 11.26 & 31.32 \pm 13.41 & 6.031(0.138) \\ \text { Post } & 41.83 \pm 4.86 & 39.19 \pm 8.90 & 37.08 \pm 12.13 & \\ \text { Post-pre } & -0.96 \pm 1.40 & -2.80 \pm 3.67 & -5.76 \pm 5.78 & 5.023(0.013)^{*} \\ t(p) & -2.282(0.045)^{*} & -2.533(0.030)^{*} & -3.333(0.008)^{* *} & \mathrm{~A} \mid \mathrm{B}, \mathrm{C}\end{array}$

The muscle activation of the rectus femoris in Devéloppé (\%MVIC)

$\begin{array}{llccc}\text { Pre } & 69.90 \pm 6.31 & 65.03 \pm 4.82 & 67.24 \pm 5.72 & 2.048(0.147) \\ \text { Post } & 63.75 \pm 10.00 & 52.45 \pm 10.42 & 56.14 \pm 6.02 & \\ \text { Post-pre } & -6.15 \pm 7.61 & -12.58 \pm 10.70 & -11.09 \pm 10.02 & 4.471(0.020)^{*} \\ t(p) & 2.680(0.023)^{*} & 3.898(0.003)^{* *} & 3.672(0.004)^{* *} & \mathrm{~A} \mid \mathrm{B}, \mathrm{C}\end{array}$

Pressure pain threshold

\begin{tabular}{lcccc} 
Pre & $25.13 \pm 7.2$ & $23.94 \pm 9.22$ & $19.18 \pm 4.65$ & $2.869(0.072)$ \\
Post & $26.88 \pm 7.5$ & $26.20 \pm 12.98$ & $21.79 \pm 5.27$ & \\
Post-pre & $1.75 \pm 2.3$ & $2.26 \pm 4.86$ & $2.61 \pm 2.41$ & $0.997(0.381)$ \\
$t(p)$ & $-2.522(0.030)^{*}$ & $-1.545(0.153)$ & $-3.586(0.005)^{*}$ & \\
\hline
\end{tabular}

${ }^{a}$ Values are expressed as mean \pm standard deviation

MET: muscle energy technique; V-A: vibration-assisted; MVIC: maximum voluntary isometric contraction; tone-way analysis of variance; ${ }^{*} p<.05 ;{ }^{* *} p<.01$ 


\section{Discussion}

Since ballet dancers produce movements greater than the normal range of motion, general objective medical assessments are not valid. This is why dynamic flexibility for movements within the maximum range of motion must be measured. Therefore, this study implemented static stretching, MET stretching and V-A stretching on the RF and compared the measurements of flexibility through the hip joint angle while in arabesque. The result showed that, MET stretching and V-A stretching are more effective for the flexibility of ballet dancers.

The suggested static stretching, MET stretching, and V-A stretching are the most widely used stretching methods in clinical practice. More specifically, the MET stretching method resists different intensity and direction applied by the therapist with voluntary contraction [17]. The basis of the hold-relax method promotes proprioceptive neuromuscular method and, aims to induce relaxation of the muscle to extend after maximal isometric contraction in static resistance and increase the range of motion and flexibility of the related muscles [18]. Yildirim, et al. [10] reported that MET stretching was more effective than static stretching when performed by 26 healthy young adults for the increase of hip joint range of motion. Feland, et al. [19] also reported that MET stretching on the hamstrings of 97 senior athletes was statistically more effective than static stretching. Similar to previously published studies, our study identified MET stretching as more effective than static stretching.

Through vibration stimulus, the muscle repeatedly contracts concentrically and eccentrically on a regular interval, placing it in an excessive gravity status [20]. Excessive gravity status stimulates the muscle spindle to activate the proprioceptors and Golgi tendon organs. This stimulates type Ia and II fibers of concentric nerve fibers to promote reflex contraction and to lead to tonic vibration reflex, which makes short and quick changes in the length of the muscle-tendon complex. As a result, this signals the sensitivity of involuntary 
muscle contraction and relaxation [21]. V-A stretching uses mechanical equipment to administer a vibration stimulus of a specific amplitude and frequency to a specific muscle. Our study utilized a frequency range of $30 \mathrm{~Hz}$, which is within the range of $20 \sim 50 \mathrm{~Hz}$ that Rittweger, et al. [22] suggested to minimize pain and induce muscle relaxation. As a result, hip joint extension angles increased more in the V-A stretching group compared to the static stretching group. This concurs with the study of Sands, et al. [23] in which the stretching method with focal vibration increased the range of motion more significantly than static stretching alone. In our study, all stretching methods showed statistically significant increases after the intervention. However, the MET stretching and the V-A stretching methods showed great increase compared to the standard procedure stretching. Although the V-A stretching group was not statistically significant compared to the MET stretching group, it showed a major difference in the increase. Based on these result, we hypothesize that the neurophysiological mechanisms combined with static stretching produced a greater effect on muscle extension.

Muscle activation, depending on the stretching method was calculated as a percentage of the MVIC value measured before the intervention on the RF; this is the main muscle for Devéloppé. As a result, after stretching, there was an $8.79 \%, 19.21 \%$, and $16.50 \%$ statistically significant decrease in muscle activation $(\mathrm{p}<0.05)$ in the static, MET and V-A stretching groups, respectfully. Previous studies report muscle activation decreases after stretching. Cramer, et al. [24] reported that measuring maximum rotation and EMG after stretching the quadriceps, decreased power production and muscle activation. Fowles, et al. [25] reported that stretching the plantar flexors results in EMG and motor unit activation decrease. Neurophysiologically, the decrease of muscle activation due to stretching is the effect on the inability of strength production due to the decrease in motor unit activation in the muscle, and from the effect of the decrease in motor neuron firing frequency due to the unknown inhibition mechanism from 
the central nervous system [26]. When lengthened to $20 \%$ more of the length in its normal state, the muscle produces maximum power. However, when it is lengthened more, the connection of cross bridge and actin is damaged causing muscle weakness [27]. Additionally, the decrease in muscle activation is caused by changes in muscle elasticity, the Golgi-tendon reflex, and the twitch force output [25]. Likewise, the significant decrease in muscle activation depending on each stretching method may result from the significant decrease of muscle activation depending on motor unit activation. More specifically, MET stretching and V-A stretching groups show statistically significant muscle activation decreases compared to the static stretching group due to a more effective stretching technique.

Finally, when we examine the pre- and post-changes of pressure pain by intervention, the static stretching group increased from $25.13 \mathrm{~N}$ to $26.88 \mathrm{~N}$ and the $\mathrm{V}-\mathrm{A}$ stretching group increased from $19.18 \mathrm{~N}$ to $21.79 \mathrm{~N}$. The MET stretching group did not demonstrate a statistically significant change. Stretching expands the reactivity of the pressure pain-sensitive pain producing the point to maximum range, allowing the deactivation for the decrease in muscle pain [28]. The additional vibration stimulus inhibits the nociceptive message in the change of pain sensation, raising the pain threshold value of the muscle [22]. Despite the study by Wilson, et al. [29] reporting that MET stretching decreases pain at lumbar and cervical levels, we were not able to produce significant pressure pain value changes in the MET stretching group. The study by Boswell [30] seems to show that a single MET method therapy results in immediate effect in pain threshold; they reported that the pressure pain threshold value of the control group was not statistically significantly different. Wilson, et al. [29] reported that a single therapy of MET stretching hardly affects the pain level, and to affect a clinically significantly difference, at least, two or more approaches are required. In our study, the reason the MET stretching group did not show a significant increase was probably that it was administered as a single 
intervention. 


\section{Conclusion}

While there are various stretching methods, there are not enough studies comparing the effects of V-A stretching, MET stretching, and static stretching. We have identified that V-A stretching is a beneficial method for flexibility, muscle activation, and pressure pain threshold in ballet dancers. However, we only observed temporary effects of V-A stretching, MET stretching, and static stretching. Therefore, future studies are need to assess the flexibility training effects on body parts using these three interventions and their carryover effects. 


\section{References}

1. Clippinger, K. Dance Anatomy and Kinesiology, 2E; Human Kinetics: 2015.

2. Kassing, G.; Jay, D.M. Teaching Beginning Ballet Technique; Human Kinetics: 1998.

3. Biernacki, J.L.; Stracciolini, A.; Griffith, K.L.; D'Hemecourt, P.A.; Owen, M.; Sugimoto, D. Association between coping skills, past injury and hip pain and function in adolescent elite female ballet dancers. Phys Sportsmed 2018, 10.1080/00913847.2018.1423853, 1-8, doi:10.1080/00913847.2018.1423853.

4. Simmons, R.W. Neuromuscular responses of trained ballet dancers to postural perturbations. Int J Neurosci 2005, 115, 1193-1203, doi:10.1080/00207450590914572.

5. Gupta, A.; Fernihough, B.; Bailey, G.; Bombeck, P.; Clarke, A.; Hopper, D. An evaluation of differences in hip external rotation strength and range of motion between female dancers and non-dancers. British journal of sports medicine 2004, 38, 778-783, doi:10.1136/bjsm.2003.010827.

6. Saito, S.; Obata, H.; Kuno-Mizumura, M.; Nakazawa, K. On the skilled plantar flexor motor action and unique electromyographic activity of ballet dancers. Exp Brain Res 2018, 236, 355-364, doi:10.1007/s00221-017-5131-0.

7. DiPasquale, S.; Wood, M. The effect of classical ballet and contemporary dance training on hip extensor flexibility and strength in novice dancers: A pilot study. Performance Enhancement \& Health $\quad$ 2017, $\quad 5, \quad 108-114$, doi:https://doi.org/10.1016/j.peh.2016.11.003.

8. Sands, W.A.; McNeal, J.R.; Jemni, M.; Penitente, G. Thinking sensibly about injury prevention and safety. Science of Gymnastics Journal 2011, 3, 43-58.

9. Sharman, M.J.; Cresswell, A.G.; Riek, S. Proprioceptive neuromuscular facilitation stretching : mechanisms and clinical implications. Sports Med 2006, 36, 929-939. 
10. Yildirim, M.S.; Ozyurek, S.; Tosun, O.; Uzer, S.; Gelecek, N. Comparison of effects of static, proprioceptive neuromuscular facilitation and Mulligan stretching on hip flexion range of motion: a randomized controlled trial. Biol Sport 2016, 33, 89-94, doi:10.5604/20831862.1194126.

11. Konrad, A.; Stafilidis, S.; Tilp, M. Effects of acute static, ballistic, and PNF stretching exercise on the muscle and tendon tissue properties. Scand J Med Sci Sports 2017, 27, 1070-1080, doi:10.1111/sms.12725.

12. Kokkonen, J.; Nelson, A.G.; Eldredge, C.; Winchester, J.B. Chronic static stretching improves exercise performance. Medicine and science in sports and exercise 2007, 39, 1825-1831, doi:10.1249/mss.0b013e3181238a2b.

13. Feland, J.B.; Hopkins, T.; Hunter, I. Acute Changes In Hamstring Flexibility Using A Wholebody-vibration Platform With Static Stretch: 2115 5: 00 PM-5: 15 PM. Medicine \& Science in Sports \& Exercise 2005, 37, S410.

14. van den Tillaar, R. Will whole-body vibration training help increase the range of motion of the hamstrings? Journal of strength and conditioning research / National Strength \& Conditioning Association 2006, 20, 192-196, doi:10.1519/R-17064.1.

15. Rittweger, J.; Beller, G.; Felsenberg, D. Acute physiological effects of exhaustive whole-body vibration exercise in man. Clin Physiol 2000, 20, 134-142.

16. Saghaei, M. Random allocation software for parallel group randomized trials. $B M C$ Med Res Methodol 2004, 4, 26, doi:10.1186/1471-2288-4-26.

17. Greenman, P.E. Principles of Manual Medicine; Lippincott Williams \& Wilkins: 2003.

18. Spernoga, S.G.; Uhl, T.L.; Arnold, B.L.; Gansneder, B.M. Duration of Maintained Hamstring Flexibility After a One-Time, Modified Hold-Relax Stretching Protocol. Journal of athletic training 2001, 36, 44-48. 
19. Feland, J.B.; Myrer, J.W.; Merrill, R.M. Acute changes in hamstring flexibility: PNF versus static stretch in senior athletes. Physical Therapy in Sport 2001, 2, 186-193, doi:https://doi.org/10.1054/ptsp.2001.0076.

20. Torvinen, S.; Kannus, P.; Sievanen, H.; Jarvinen, T.A.; Pasanen, M.; Kontulainen, S.; Nenonen, A.; Jarvinen, T.L.; Paakkala, T.; Jarvinen, M., et al. Effect of 8-month vertical whole body vibration on bone, muscle performance, and body balance: a randomized controlled study. Journal of bone and mineral research : the official journal of the American Society for Bone and Mineral Research 2003, 18, 876-884, doi:10.1359/jbmr.2003.18.5.876.

21. Cardinale, M.; Lim, J. Electromyography activity of vastus lateralis muscle during whole-body vibrations of different frequencies. Journal of strength and conditioning research / National Strength \& Conditioning Association 2003, 17, 621-624.

22. Rittweger, J.; Just, K.; Kautzsch, K.; Reeg, P.; Felsenberg, D. Treatment of chronic lower back pain with lumbar extension and whole-body vibration exercise: a randomized controlled trial. Spine 2002, 27, 1829-1834.

23. Sands, W.A.; McNeal, J.R.; Stone, M.H.; Russell, E.M.; Jemni, M. Flexibility enhancement with vibration: Acute and long-term. Medicine and science in sports and exercise 2006, 38, 720 .

24. Cramer, J.T.; Housh, T.J.; Weir, J.P.; Johnson, G.O.; Coburn, J.W.; Beck, T.W. The acute effects of static stretching on peak torque, mean power output, electromyography, and mechanomyography. European journal of applied physiology 2005, 93, 530-539, doi:10.1007/s00421-004-1199-x.

25. Fowles, J.R.; Sale, D.G.; MacDougall, J.D. Reduced strength after passive stretch of the human plantarflexors. J Appl Physiol (1985) 2000, 89, 1179-1188. 
26. Behm, D.G.; Button, D.C.; Butt, J.C. Factors affecting force loss with prolonged stretching. Canadian journal of applied physiology $=$ Revue canadienne de physiologie appliquee 2001, 26, 261-272.

27. Winter, D.A. Biomechanics and motor control of human movement; John Wiley \& Sons: 2009.

28. Hanten, W.P.; Olson, S.L.; Butts, N.L.; Nowicki, A.L. Effectiveness of a home program of ischemic pressure followed by sustained stretch for treatment of myofascial trigger points. Physical therapy 2000, 80, 997-1003.

29. Wilson, E.; Payton, O.; Donegan-Shoaf, L.; Dec, K. Muscle energy technique in patients with acute low back pain: a pilot clinical trial. The Journal of orthopaedic and sports physical therapy 2003, 33, 502-512, doi:10.2519/jospt.2003.33.9.502.

30. Boswell, C. The effect of a muscle energy stretch on suboccipital tenderness. Vcitoria University, 2005. 